\title{
Dynamical Behaviours of Prey-predator Fishery Model with Harvesting Affected by Toxic Substances
}

\author{
Ang Tau Keong*, Hamizah M Safuan and Kavikumar Jacob \\ Department of Mathematics and Statistics, Faculty of Science, Technology and Human Development \\ Universiti Tun Hussein Onn Malaysia, 86400 Parit Raja, Malaysia \\ ${ }^{*}$ Corresponding author: taukeong1018@gmail.com
}

Article history

Received: 15 July 2017

Received in revised form: 13 November 2017

Accepted: 27 December 2017

Published on line: 1 June 2018

\begin{abstract}
In this paper we consider a harvesting model of predator-prey fishery in which the prey is directly infected by some external toxic substances. The toxic infection is indirectly transmitted to the predator during the feeding process. The model is a modified version from the classic Lotka-Volterra predator-prey model. The stability and bifurcation analyses are addressed. Numerical simulations of the model are performed and bifurcation diagrams are studied to investigate the dynamical behaviours between the predator and the prey. The effects of toxicity and harvesting on the stability of steady states found in the model are discussed.
\end{abstract}

Keywords Harvesting; fishery; toxicant; predator; prey.

Mathematics Subject Classification 92B08.

\section{Introduction}

In the recent decades, human desire seems to be profound and even endless. Due to the global modernisation and urbanisation, human tend to fulfill their material cravings in various ways. This scenario foments the increasing human demands in searching more food and resources. Unfortunately, irresponsible actions and inconsiderate exploitation on natural resources such as fisheries and forestry will result in some unpleasant effects on the environment.

One of the most significant problems is the effects of toxicants on the dynamics of ecosystem. A toxicant is defined as any anthropogenic toxic substance released into environment through human activities. Unlike toxicant, a toxin is a poisonous substance that naturally produced within an organism. Recently, many research have been done to investigate the impacts of toxicants on the ecosystem health. Some of the leading studies are the work of Hallam and Clark [1] which emphasised on the effects on the first order kinetics of a population in the presence of toxicity. They proposed a threshold value of terminal organismal concentration that can control the extinction rate of the population even in the limiting toxicants condition. Further work on the presence of toxicity was done by Hallam and De Luna [2] where they 
modified the model by taking into account of toxicity from both environmental and food chain pathways.

Other work regarding the effects of toxicants on general single species was done by Freedman and Shukla [3]. The steady states of the prey-predator system were found, and the amount of toxicants keep reducing at a certain rate. The impacts of toxicants on two species competing with each other were studied by Chattopadhyay [4] where the presence of toxicants was found to be an important element for the persistence of the population. Huang et al. [5] modified the model of prey-predator with the influence of environmental toxicants where both prey and predator are exposed to the toxicants simultaneously. However, most of the models dealing with toxicants are limited to general communities but not specific to a certain aquatic environment.

Many researchers now have started to study the mathematical models dealing with toxicants in fishery perspectives. The model developed by Huang et al. [6] considered both toxins in the population and also in the environment. The complex dynamical behaviours of fish population in the presence of toxicants was discussed by Scott and Sloman [7] where the reproduction growth, social behaviours and hormone production of fish were observed.

Kar and Chaudhuri [8] studied a model which combined both underlying concepts of harvesting term and toxicity in two competing fish species. They found that the persistence and extinction properties of a large marine ecosystem are not only dependent on the inter specific interaction between the marine species but it also dependent of the external environment factors. Slightly different from the work of Kar and Chaudhuri [8], Das et al. [9] incorporated the effects of toxicants in a prey-predator fishery model in which the growths of both prey and predator species vary at different rates.

The main objective of the present paper is to study the dynamical properties of a preypredator fishery model in the presence of toxicity. Both prey and predator species are subjected to a certain rate of harvesting. The rate of exposure to the toxicants is different for both species. We study the effects of toxicants on both prey and predator species to make a further conjecture on the persistence and extinction properties.

\section{Model Formulation}

Generally, the form of the classical prey-predator model, which is commonly known as LotkaVolterra model [10] is

$$
\begin{aligned}
& \frac{d X}{d t}=r_{1} X-\gamma_{1} X Y \\
& \frac{d Y}{d t}=-r_{2} Y+\gamma_{2} X Y
\end{aligned}
$$

where the prey, $X$, grows at a rate of $r_{1}$ in the absence of predator, $Y$. Similarly, the predator dies out at a rate of $r_{2}$ in the absence of prey. Parameters $\gamma_{1}$ and $\gamma_{2}$ represent the respective rates of change of prey and predator due to the prey-predation. The prey population grows exponentially in the absence of predator which is unreasonable [11]. In order to overcome this 
problem, the logistic limiting term is added to the prey population and we have the system of

$$
\begin{aligned}
& \frac{d X}{d t}=r_{1} X\left(1-\frac{X}{K}\right)-\gamma_{1} X Y \\
& \frac{d Y}{d t}=-r_{2} Y+\gamma_{2} X Y
\end{aligned}
$$

where $K$ is the environmental carrying capacity of the prey population. The model assumes that the prey population grows logistically with the rate of $r_{1}$ in the absence of predator while the predator population dies out exponentially with a rate of $r_{2}$ in the absence of prey. Since our main objective is to examine the dynamical behaviours of prey-predator fishery in the presence of harvesting and toxicity, we study the system [9]

$$
\begin{aligned}
& \frac{d X}{d t}=r_{1} X\left(1-\frac{X}{K}\right)-\gamma_{1} X Y-a E X-b X^{3}, \\
& \frac{d Y}{d t}=-r_{2} Y+\gamma_{2} X Y-c E Y-d Y^{2},
\end{aligned}
$$

where the catchability coefficients of the prey and predator population are denoted by $a$ and $c$, respectively. Both populations are subjected to a combined harvesting effort, $E$, and the catch rate function of $a E X$ and $c E Y$ follow the catch-per-unit-effort rules. In this model, the prey fish species is directly infected by environmental toxicants such as the industrial waste and the predator fish species is indirectly infected by the toxicants through the feeding process on the infected prey species. Parameters $b$ and $d$ denote the coefficients of toxicity on prey and predator, respectively. All parameters are assumed to be positive values.

\section{$2.1 \quad$ Non-dimensional Model}

In order to reduce the number of parameters for better interpretation and computation, the non dimensionalisation of system (3) is carried out using a set of scaled variables

$$
x=\frac{\gamma_{2} X}{r_{1}}, y=\frac{\gamma_{1} Y}{r_{1}}, \tau=r_{1} t .
$$

The dimensional system (3) becomes

$$
\begin{aligned}
& \frac{d x}{d \tau}=x(1-\alpha x)-x y-g_{1} x-h_{1} x^{3}, \\
& \frac{d y}{d \tau}=-\beta y+x y-g_{2} y-h_{2} y^{2}
\end{aligned}
$$

where

$$
\alpha=\frac{r_{1}}{\gamma_{2} K}, g_{1}=\frac{a E}{r_{1}}, h_{1}=\frac{b r_{1}}{\left(\gamma_{2}\right)^{2}}, \beta=\frac{r_{2}}{r_{1}}, g_{2}=\frac{c E}{r_{1}}, h_{2}=\frac{d}{\gamma_{1}} .
$$

In the system (4),

$\alpha$ : ratio of the growth rate of $x$ to the product of growth rate of $y$ by $x$ and environmental carrying capacity of $x$;

$g_{1}$ : ratio of the product of catchability coefficient of $x$ and harvesting effort to the growth 
rate of $x$;

$h_{1}$ : ratio of the product of coefficient of toxicity and growth rate of $x$ to the square of growth rate of $y$ by $x$;

$\beta$ : ratio of the death rate of $y$ to the growth rate of $x$;

$g_{2}$ : ratio of the product of catchability coefficient of $y$ and harvesting effort to the growth rate of $x$;

$h_{2}$ : ratio of the coefficient of toxicity on $y$ to the death rate $x$ by $y$.

\section{Steady States, Equilibria and Stability Analysis}

There are four possible steady states of $P_{i}$ in the form of $(x, y)$ where $i$ represents the number of steady states occur in the system and three of them are trivial steady states:

$$
P_{0}=\left(0,-\frac{\beta+g_{2}}{h_{2}}\right), \quad \mathrm{P}_{1}=(0,0) \quad \text { and } \quad P_{2}=(\tilde{x}, 0)
$$

where $\tilde{x}$ is the root of the quadratic equation of

$$
h_{1} \tilde{x}^{2}+\alpha \tilde{x}+g_{1}-1=0 \quad \text { or } \quad \tilde{x}=\frac{-\alpha+\sqrt{\alpha^{2}-4 h_{1}\left(g_{1}-1\right)}}{2 h_{1}} .
$$

In the present paper, the steady state of $P_{0}$ is not considered since $-\frac{\beta+g_{2}}{h_{2}}$ represents the negative population size which is not biologically meaningful. The equilibrium of $P_{1}$ represents when both the prey and predator population die out and extinct. It has the characteristic equation of

$$
\lambda^{2}+\left(g_{1}+g_{2}+\beta-1\right) \lambda+\left(g_{1}-1\right)\left(g_{2}+\beta\right)=0,
$$

where $\lambda$ is the eigenvalue. Equilibrium $P_{1}$ has a set of eigenvalues of $E_{P_{1}}=\left\{1-g_{1},-\beta-g_{2}\right\}$. Note that since $-\beta-g_{2}$ is always negative, thus $P_{1}$ is a stable node if $g_{1}>1$ or $P_{1}$ is an unstable saddle point if $g_{1}<1$.

On the other hand, the equilibrium of $P_{2}$ represents when the prey population survives in the absence predator population. It has the characteristic equation of

$$
\lambda^{2}+\left(3 \tilde{x}^{2} h_{1}+2 \alpha \tilde{x}+\beta-\tilde{x}+g_{1}+g_{2}-1\right) \lambda+\left(3 \tilde{x}^{2} h_{1}+2 \alpha \tilde{x}+g_{1}-1\right)\left(\beta-\tilde{x}+g_{2}\right)=0,
$$

with a set of eigenvalues $E_{P_{2}}=\left\{-\beta+\tilde{x}-g_{2},-3 \tilde{x}^{2} h_{1}-2 \alpha \tilde{x}-g_{1}+1\right\}$, where $\tilde{x}$ is given in (5).

The non-trivial steady state (coexistence equilibrium) of the model (4) is $P_{3}=\left(\hat{x}, \frac{\hat{x}-\beta-g_{2}}{h_{2}}\right)$ where $\hat{x}$ is obtained by solving the quadratic equation

$$
c_{1} \hat{x}^{2}+c_{2} \hat{x}+c_{3}=0
$$

with

$$
c_{1}=h_{1} h_{2}, \quad c_{2}=\alpha h_{2}+1 \quad \text { and } \quad c_{3}=h_{2}\left(g_{1}-1\right)-\beta-g_{2},
$$

and

$$
\hat{x}=\frac{-\left(\alpha h_{2}+1\right)+\sqrt{\left(\alpha h_{2}+1\right)^{2}-4 h_{1} h_{2}\left(g_{1} h_{2}-h_{2}-\beta-g_{2}\right)}}{2 h_{1} h_{2}} .
$$




\section{Bifurcation Results and Analysis}

We investigate the dynamical behaviours of the prey-predator fishery with toxicity by performing bifurcation analysis using parameter variation [12]. In order to examine the steady states for further interpretation, the model (4) is analysed by using the numerical software of XPPAUT and the steady state diagrams are obtained using the MATLAB software. For simplicity, we set the parameter of $\alpha=0.1, g_{1}=0.1, h_{1}=0.5, \beta=0.5, g_{2}=0.5$ and $h_{2}=1$. In this section, we investigate the effects of the toxicant parameter $\left(h_{1}\right)$ found in the system (4). Figure (1a) and $(1 \mathrm{~b})$ illustrate the steady state diagrams with respect to the toxicant parameter, $h_{1}$. In both diagrams, the blue solid lines represent the stable steady states while the red dotted lines represent the unstable steady states.

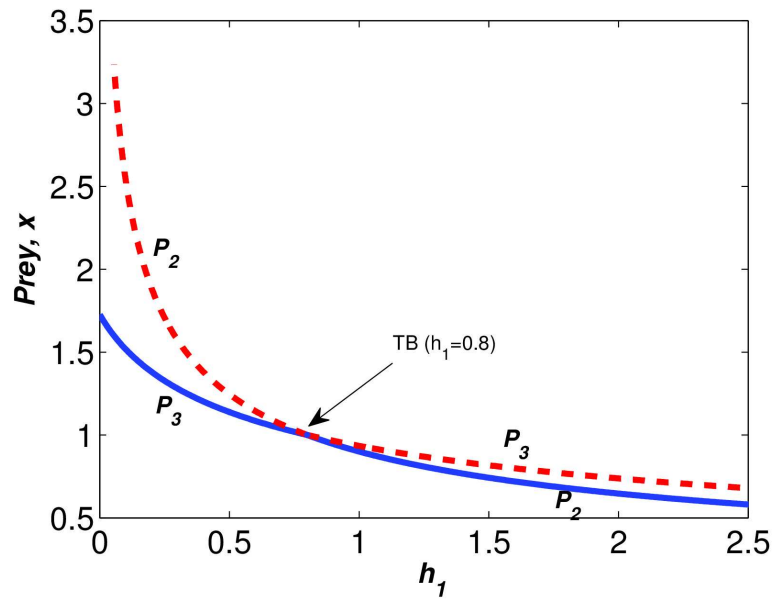

(a)

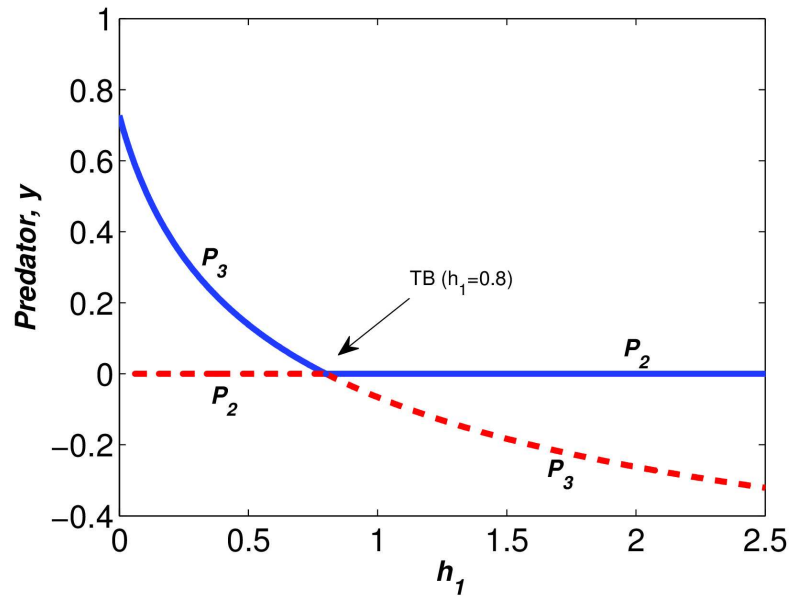

(b)

Figure 1: Steady-state Diagrams of Model (4) with respect to the Toxicant Parameter, $h_{1}$, with $\alpha=0.1, g_{1}=0.1, \beta=0.5, g_{2}=0.5$ and $h_{2}=1$ for (a) Prey, $x$, and (b) Predator, $y$, respectively

Referring to the Figure (1a) and (1b), the transcritical bifurcation (TB) occurs where the two steady-state branches of $P_{2}$ and $P_{3}$ interchange with each other after passing through a transcritical bifurcation point. From the diagrams, the population densities of both $x$ (prey) and $y$ (predator) decrease as the toxicant parameter of $h_{1}$ increases. This scenario happens because the presence of toxicants affects the growth of prey and consequently depletes the number of predator which depends on the prey as a food source. This scenario is described by the solid lines of $P_{3}$ in Figure (1a) and (1b). On the other hand, as the toxicant parameter of $h_{1}$ increasing until it passes through a transcritical bifurcation point $\left(h_{1}=0.8\right)$ the two steady-state branches interchange where the steady state $P_{3}$ becomes unstable and $P_{2}$ becomes stable. In results, the population density of the prey continues to decrease, however, the predator population dies off and finally extincts due to the high level of toxicants. The predator population decreases more drastically than prey because the presence of toxicants will not only affect the predator indirectly, but also reduces the food source of predator population. In short, if the toxicant 
parameter $h_{1}<0.8$, the coexistence of prey and predator occurs but if $h_{1}>0.8$, the predator species dies off.

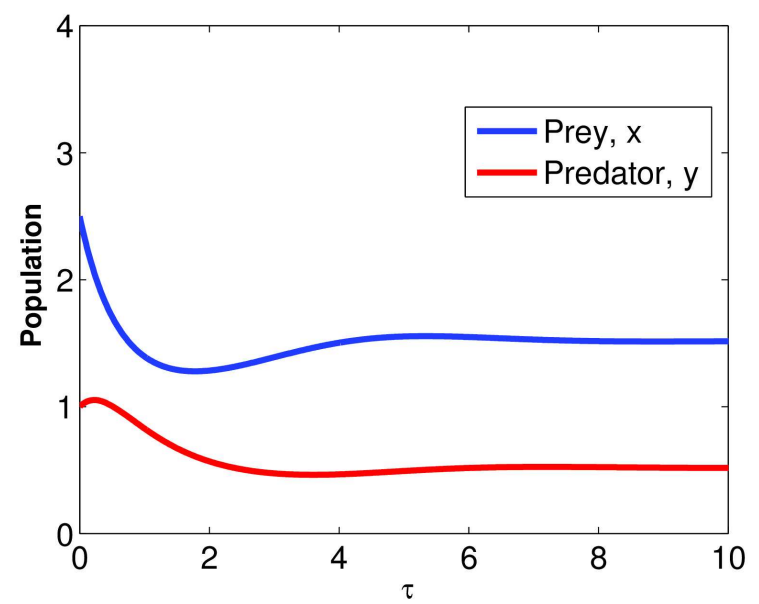

(a)

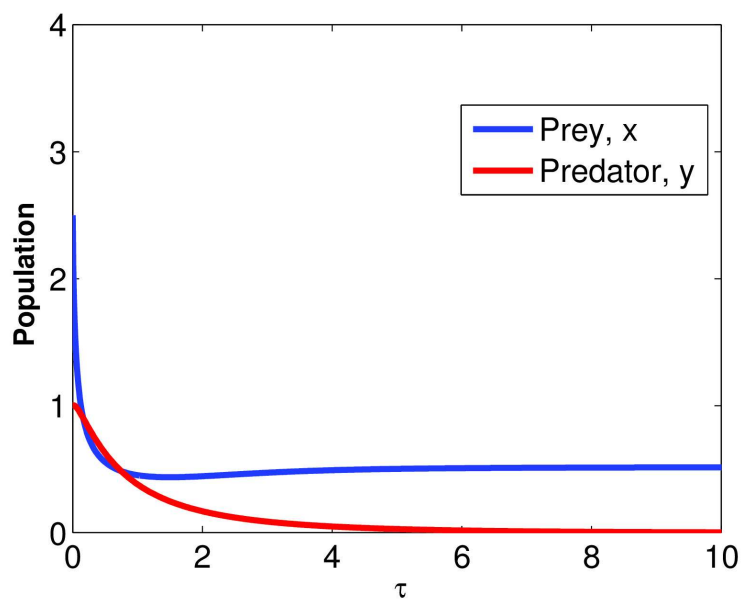

(b)

Figure 2: Time Plots of Model (4) with $\alpha=0.1, g_{1}=0.1, \beta=0.5, g_{2}=0.5, h_{2}=1$ and Initial Conditions of $\left(x_{0}, y_{0}\right)=(2.5,1)$ at (a) $h_{1}=0.1$ and (b) $h_{1}=3.2$ respectively

In order to observe the underlying trends and behaviours over time of the population in system $(4$,$) time series plots are obtained using MATLAB software as shown in the Figure (2a)$ and (2b), using the same values of parameters and by fixing the value of $h_{1}=0.1$ and $h_{1}=3.2$. For Figure (2a), the value of toxicant parameter is $h_{1}<0.8$, where coexistence of the both species $x$ and $y$. In Figure $(2 \mathrm{~b},) h_{1}>0.8$, the extinction of the predator $y$ occurs due to the loss of food source and infection of toxicants. In Figure 2a, the population of prey decreases slightly at the beginning before they increase to a maximum value and reach the stable steady state values. This scenario is due to the predation activities conducted by the predator on the prey. The predator population continues to decrease until they are eliminated from the system, however, the prey population continue to persist.

Besides investigating the dynamical behaviours of prey-predator fishery with respect to the toxicant parameter, we are also interested in the effects of the harvesting parameter, $g_{2}$ of the model (4). Figure (3a) and (3b) depict the steady state diagrams of model (4) with respect to the harvesting parameter of $g_{2}$.

From Figure (3a) and (3b), the prey population $x$ increases but the predator population $y$ decreases as the harvesting parameter, $g_{2}$ increases before passing through the transcritical bifurcation point of $g_{2}=0.7454$. This is because the increasing of harvesting activities on predator suppress the predator population and consequently reduce the predation activities on the prey population. This scenario is described by the blue solid lines of $P_{3}$ where the coexistence of the both prey and predator population occurs. On the contrary, the extinction of the predator population occurs when the harvesting parameter $g_{2}>0.7454$ which is denoted by the blue solid lines labelled as $P_{2}$.

From Figure (4a) and (4b), when the harvesting activities on the predator are maintained 


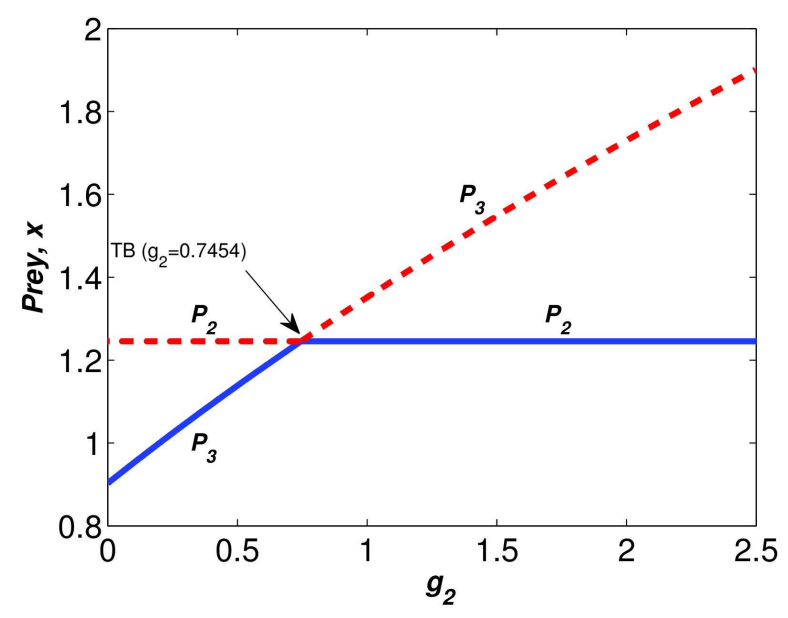

(a)

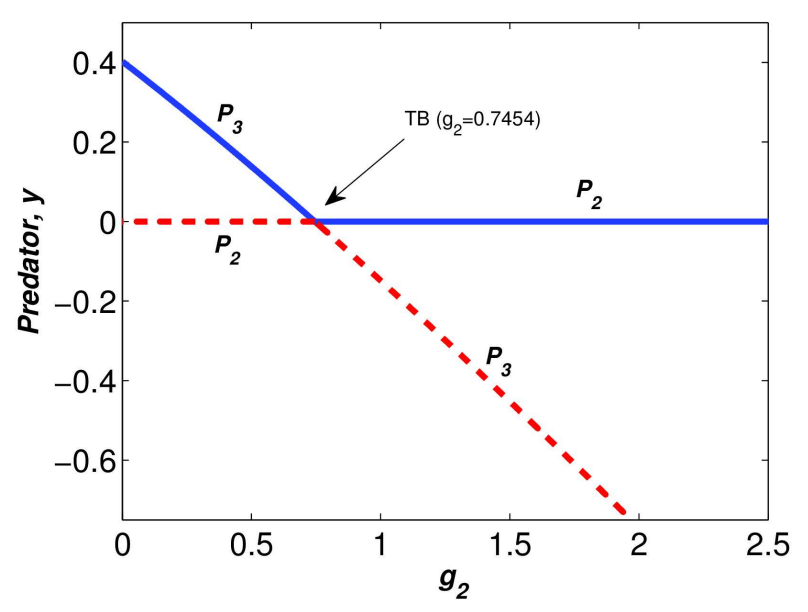

(b)

Figure 3: Steady-state Diagrams of Model (4) with respect to the Harvesting Parameter of $g_{2}$ with $\alpha=0.1, g_{1}=0.1, h_{1}=0.5, \beta=0.5$ and $h_{2}=1$ for (a) Prey, $x$ and (b) Predator, $y$, respectively

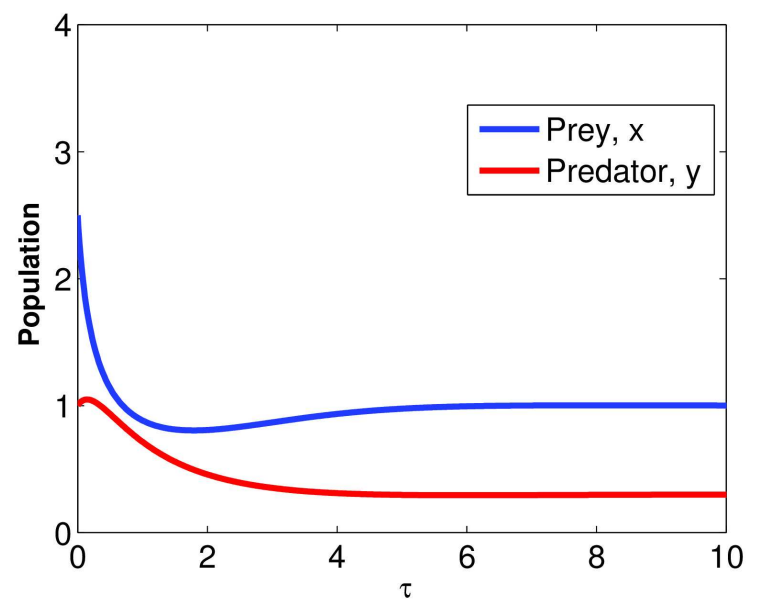

(a)

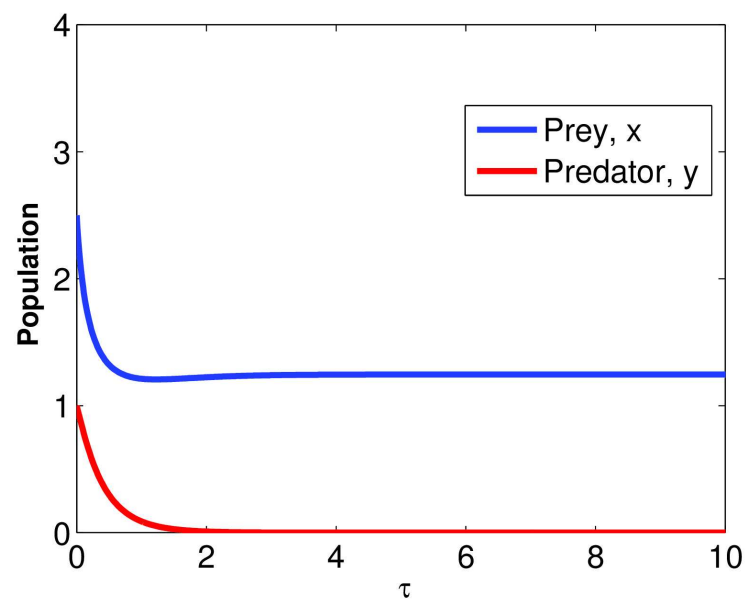

(b)

Figure 4: Time Plots of Model (4) with $\alpha=0.1, g_{1}=0.1, h_{1}=0.5 \beta=0.5, h_{2}=1$ and Initial Conditions of $\left(x_{0}, y_{0}\right)=(2.5,1)$ at (a) $g_{2}=0.2$ and (b) $g_{2}=3$ respectively

below the threshold level, 0.7454 both prey and predator population survive and persist together. Excessive harvesting activities on predator will lead to the extinction of the predator population and only the prey population can persist. 


\section{Conclusion}

In the previous sections, a prey-predator system of a fishery model by considering the influence of toxicants and harvesting activities has been investigated. The toxicants affect the prey species directly while the predator species get infected indirectly through the feeding process. Harvesting process is conducted on both prey and predator population with similar harvesting effort.

The effects of toxicants and harvesting efforts to prey-predator model is observed with different values of the toxicant parameter, $h_{1}$ and harvesting parameter, $g_{2}$ that showed the impacts on the prey and predator population. From the steady state diagrams, low level of toxicants and harvesting activities leads to the coexistence of both species but conversely, high level of toxicants and harvesting activities eradicates the entire predator population in a short period of time. Moreover, from the time series plots, we can observe that the prey population continues to survive and persists even at a high level of toxicants since the prey follows the logistic growth compared to the predator.

Therefore, rational initiatives should be conducted to ensure that both toxicants and harvesting levels are in moderate levels in order to protect the entire aquatic ecosystem from collpase.

\section{Acknowledgements}

The present research is supported by the Fundamental Research Grant Scheme, Ministry of Higher Education Malaysia Vot number 1563 and Universiti Tun Hussein Onn Malaysia.

\section{References}

[1] Hallam, T. G. and Clark, C. E. Effects of toxicants on populations: A qualitative approach I. Equilibrium environmental exposure. Ecological Modelling. 1983. 18: 291-304.

[2] Hallam, T. G. and De Luna, J. T. Effects of toxicants on populations: A qualitative approach III. Environmental and food chain pathways. J. Theor. Biol. 1984. 109: 411429.

[3] Freedman, H. I. and Shukla, J. B. Models for the effect of toxicant in single-species and predator-prey systems. J. Math. Biol. 1991. 30: 15-30.

[4] Chattopadhyay, J. Effect of toxic substances on a two-species competitive system. Ecological Modelling. 1996. 84: 287-289.

[5] Huang, Q., Wang, H. and Lewis, Mark A. The impact of environmental toxins on predatorprey dynamics. J. Theor. Biol. 2015. 378: 12-30.

[6] Huang, Q., Parshotam, L., Wang, H. and Lewis, Mark A. A model for the impact of contaminants on fish population dynamics. J. Theor. Biol. 2013. 334: 71-79. 
[7] Scott, G. R. and Sloman, K. A. Effects of environmental pollutant on complex fish behaviour: intergrating behavioural and physiological indicators of toxicity. Aquatic Toxicology. 2004. 68: 369-392.

[8] Kar, T. K. and Chaudhuri, K. S. On non-selective harvesting of two competing fish species in the presence of toxicity. Ecological Modelling. 2003. 161: 125-137.

[9] Das, T., Mukherjee, R. N. and Chaudhuri, K. S. Harvesting of a prey-predator fishery in the presence of toxicity. Applied Mathematical Modelling. 2009. 33: 2282-2292.

[10] Jang, S. RJ. On the Lotka-Volterra competition system with Allee effects. Comp. Appl. Math.. 2013. 32: 179-189.

[11] Berryman, Alan A. The origins and evolution of predator-prey theory. Ecology. 1992. 73(5): 1530-1535.

[12] Crawford, J. D. Introduction to bifurcation theory. Rev. Mod. Phys.. 1991. 63(4): 991-1037. 